\title{
Sophie Arnaud, La voix de la nature dans l'œuvre de Jacques Peletier du Mans (1517-1582)
}

\section{Dario Cecchetti}

\section{(2) OpenEdition}

1 Journals

\section{Edizione digitale}

URL: http://journals.openedition.org/studifrancesi/27422

DOI: 10.4000/studifrancesi.27422

ISSN: 2421-5856

\section{Editore}

Rosenberg \& Sellier

\section{Edizione cartacea}

Data di pubblicazione: 31 décembre 2006

Paginazione: 592

ISSN: 0039-2944

\section{Notizia bibliografica digitale}

Dario Cecchetti, «Sophie Arnaud, La voix de la nature dans l'œuvre de Jacques Peletier du Mans

(1517-1582) », Studi Francesi [Online], 150 (L | III) | 2006, online dal 30 novembre 2015, consultato il 08 novembre 2020. URL : http://journals.openedition.org/studifrancesi/27422 ; DOI : https://doi.org/ 10.4000/studifrancesi.27422

\section{Questo documento è stato generato automaticamente il 8 novembre 2020}

\section{(c) (1)}

Studi Francesi è distribuita con Licenza Creative Commons Attribuzione - Non commerciale - Non opere derivate 4.0 Internazionale. 


\title{
Sophie Arnaud, La voix de la nature dans l'œuvre de Jacques Peletier du Mans (1517-1582)
}

\author{
Dario Cecchetti
}

\section{NOTIZIA}

SOPHIE ARNAUD, La voix de la nature dans l'œuvre de Jacques Peletier du Mans (1517-1582), Paris, Champion («Bibliothèque Littéraire de la Renaissance», 54), 2005, pp. 691.

1 Frutto di una thèse de doctorat, il lavoro che Sophie Arnaud mette a disposizione degli studiosi è un'ampia sintesi che ricostruisce l'itinerario intellettuale di Peletier, il suo pensiero, i suoi molteplici interessi culturali. L'opera di Peletier, nel suo insieme, denuncia interessi di poligrafo, pur essendo profondamente coerente: dal canzoniere petrarchista, L'Amour des Amours, vera summa del neoplatonismo cinquecentesco, a poemetti descrittivi come La Savoye, che nel celebrare natura e paesaggio sono attenti ai fenomeni della physis, noi ritroviamo non solo un'attenzione ai grandi (e svariati) modelli rinascimentali della poesia filosofica o filosoficheggiante, ma anche l'idea che la creazione è retta da un sistema organizzativo, in cui l'essenza matematica della natura conferisce al numero e all'unità un carattere fondatore. Umanista, grammatico, filosofo e matematico, Peletier ritiene (e lo teorizza nel suo Art poétique del 1555) che l'attività poetica trovi la sua legittimazione nel disvelamento della natura e che lo stesso entusiasmo poetico possa essere considerato una tappa dell'attività scientifica. Nella ricca bibliografia su Peletier comparivano parecchi studi sulla "poesia scientifica» del nostro autore, ma mancava un lavoro organico, ampio e approfondito, su quella che potremmo considerare a un tempo filosofia della natura, teologia naturale e interpretazione della realtà fenomenica alla luce delle conoscenze matematiche. Ora, questo lavoro è stato portato a termine da Sophie Arnaud, la quale, nella prima parte della sua indagine ha illustrato come, secondo Peletier, «il senso della natura organizzi la vita degli uomini secondo fondamenti matematici e la sua armonia proceda dalle 
figure disegnate dagli astri. Sia che esamini il cielo o che analizzi la serie delle cause e degli effetti, l'individuo trova al di fuori di lui la manifestazione di un ordine su cui non ha presa reale. L'intelletto non rende il mondo intelligibile: al contrario, il principio di razionalità è insito già in modo latente nel seno delle cose. È compito soltanto dell'essere umano sapere osservare e udire, essere capace di comprendere la voce della natura. Prodotto troppo umano per non dare esca all'errore e all'opinione, il linguaggio non appare qui come il privilegio che segna l'eccellenza dell'intelligenza. In rivincita, la sua missione più alta consiste nel manifestare l'ordine del mondo. [...] Nella seconda parte dell'indagine si dimostra che Peletier giunge a elaborare una 'parola naturale' che, senza cristallizzarsi nel testo e senza perdersi nelle chimere dell'opinione, riesce a restituire la dinamica dell'oralità» (p. 18). Lavoro dunque di grande impegno, quello di $\mathrm{S}$. Arnaud, utile anche allo studioso di spiritualità, teologia e filosofia rinascimentale. 